\title{
Knockout RAGE alleviates cardiac fibrosis through repressing endothelial-to-mesenchymal transition (EndMT) mediated by autophagy
}

Lu Zhang ${ }^{1,2,3}$, Jiaqi He $\mathrm{e}^{1,2,3}$, Junyan Wang $\mathbb{1}^{1,2,3}$, Jing Liu ${ }^{1,2}$, Zixin Chen ${ }^{1,2,3,4}$, Bo Deng ${ }^{1,2,3}$, Lan Wei ${ }^{1,2,3}$, Hanqin Wu ${ }^{1,2,3}$, Birong Liang ${ }^{1,2,3}$, Huan Li $\mathrm{i}^{1,2,3,4}$, Yusheng Huang ${ }^{1,2,3,4}$, Lu Lu (1) ${ }^{1,2,3,4}$, Zhongqi Yang 1,2,3,4,5, Shaoxiang Xian 1,2,3,4,5 and Lingjun Wang (1) 1,2,3,5

\begin{abstract}
Endothelial-to-mesenchymal transition (EndMT) has been shown to contribute to cardiac fibrosis and heart failure (HF). Recent studies have demonstrated that EndMT is regulated by autophagy, and we previously showed suppression of excessive autophagy and alleviation of cardiac fibrosis in HF mice with inactivated receptor for advanced glycation end products (RAGE). Thus, we investigated whether reduced cardiac fibrosis due to RAGE knockout occurred by inhibiting EndMT mediated by excessive autophagy. We found a decrease in endothelial cells $\left(\mathrm{CD} 31^{+} \mathrm{NE}\right.$-Cadherin $\left.{ }^{+}\right)$ and an increase in cells co-expressing CD31 and a-smooth muscle actin (a-SMA, myofibroblast marker) at 8 weeks in heart tissue of mice subjected to transverse aortic constriction (TAC), which implied EndMT. Knockout RAGE decreased EndMT accompanied by decreased expression of autophagy-related proteins (LC3BII/I and Beclin 1), and alleviated cardiac fibrosis and improved cardiac function in TAC mice. Moreover, 3-methyladenine (3-MA) and chloroquine (CQ), inhibitors of autophagy, attenuated EndMT, and cardiac fibrosis in TAC mice. Importantly, EndMT induced by AGEs could be blocked by autophagy inhibitor in vivo and in vitro. These results suggested that AGEs/RAGE-autophagyEndMT axis involved in the development of cardiac fibrosis and knockout RAGE ameliorated cardiac fibrosis through decreasing EndMT regulated by autophagy, which could be a promising therapeutic strategy for HF.
\end{abstract}

\section{Introduction}

Heart failure (HF) occurs as the final stage of many cardiovascular diseases and is a major health challenge worldwide. Cardiac remodeling is the process of structural and functional changes in the left ventricle and is associated with hypertrophy and apoptosis of cardiomyocytes, particularly ventricular fibrosis ${ }^{1}$, which is pivotal in developing $\mathrm{HF}^{2,3}$. The ventricular fibrotic process is initially triggered by the differentiation of cardiac fibroblasts into myofibroblasts. These activated fibroblasts

\footnotetext{
Correspondence: Lingjun Wang (smu868@163.com)

${ }^{1}$ The First Affiliated Hospital, Guangzhou University of Chinese Medicine, Guangzhou 510405, China

${ }^{2}$ The First Clinical Medical School, Guangzhou University of Chinese Medicine, Guangzhou 510405, China

Full list of author information is available at the end of the article

Edited by S. Lavandero
}

display increased proliferative capacity and secrete large amounts of extracellular matrix $(\mathrm{ECM})^{4,5}$. Resident proliferation of fibroblasts was traditionally considered as the origin of myofibroblasts ${ }^{2-5}$, but substantial evidence indicates myofibroblasts may also originate from other cellular sources. In many fibrotic conditions, myofibroblasts may stem from endothelial cells through endothelial-to-mesenchymal transition $(\text { EndMT })^{6-9}$. EndMT involves an intricate cellular differentiation process in which endothelial cells detach and migrate away from the endothelium, lose endothelial properties, and acquire mesenchymal features ${ }^{10}$. An increasing number of studies indicate EndMT is a common and potentially disease-causing process ${ }^{11-15}$ and contributes to cardiac fibrosis ${ }^{16}$. mRNA levels of EndMT-related genes are increased in the human fibrotic heart tissue ${ }^{17}$, and

\section{(c) The Author(s) 2021}

(c) (i) Open Access This article is licensed under a Creative Commons Attribution 4.0 International License, which permits use, sharing, adaptation, distribution and reproduction cc. in any medium or format, as long as you give appropriate credit to the original author(s) and the source, provide a link to the Creative Commons license, and indicate if changes were made. The images or other third party material in this article are included in the article's Creative Commons license, unless indicated otherwise in a credit line to the material. If material is not included in the article's Creative Commons license and your intended use is not permitted by statutory regulation or exceeds the permitted use, you will need to obtain permission directly from the copyright holder. To view a copy of this license, visit http://creativecommons.org/licenses/by/4.0/. 
repression of EndMT attenuates isoproterenol-induced cardiac fibrosis ${ }^{18}$. Therefore, understanding the mechanism of EndMT is essential to potentially prevent cardiac fibrosis and HF.

EndMT is regulated by autophagy ${ }^{19-21}$, which plays an important role in many physiological and pathological processes $^{22,23}$. Autophagy can be divided into three types in general. Macroautophagy is the most important, and is initiated by the formation of the phagophore, followed by the completion of a double-membrane autophagosome, then, autolysosome is formed through autophagosome dock and fuse with the lysosome, finally, acid hydrolases inside the autolysosome break down and degrade the autophagosome inner membrane and cargo ${ }^{24}$. Autophagy has been considered as a protective mechanism ${ }^{25}$ and is critical for the survival of cardiomyocytes subjected to stress and hypoxia ${ }^{26-28}$. Nevertheless, excessive autophagy is deleterious ${ }^{19}$ and accentuates cardiac fibrosis ${ }^{29}$. The increased autophagy is detrimental during reperfusion that causes significant cardiac injury ${ }^{27}$. We previously showed that excessive autophagy could be inhibited by deletion of the receptor for advanced glycation end products (RAGE), resulting in amelioration of cardiac fibrosis in $\mathrm{HF}^{30}$.

RAGE is the receptor of advanced glycation end products (AGEs). Increased AGEs and RAGE activate autophagy-associated signal pathways and induce autophagy in various diseases ${ }^{30-33}$. Recently, AGEs and their soluble receptor (sRAGE) have been confirmed as poor prognostic biomarkers of HF in non-diabetic patients ${ }^{34}$. AGEs/RAGE can also transduce fibrotic pathways ${ }^{30,35}$. Additionally, AGEs are triggers in EndMT, and AGEs/ RAGE mediated EndMT in human endothelial cells ${ }^{36}$. Therefore, we aimed to explore whether the reduction of cardiac fibrosis by knockout RAGE occurred through inhibition of autophagy-regulated EndMT in HF mice induced by transverse aortic constriction (TAC).

\section{Materials and methods \\ Animals}

All experimental animal protocols for this study were approved by the Animal Care Committee of Guangzhou University of Chinese Medicine and were strictly in accordance with the National Institutes of Health Guidelines for Care and Use of Laboratory Animals.

Eight-week-old C57BL/6J wild-type male mice weighing 18-22 $\mathrm{g}$ were obtained from the Laboratory Animal Center of Guangzhou University of Chinese Medicine. RAGE knockout mice were a gift from Kanazawa University, Japan ${ }^{37}$. Mice were randomly assigned into eight groups: Sham, Sham + AGEs, Sham + $\mathrm{RAGE}^{-1-}$, TAC, $\mathrm{TAC}+\mathrm{AGEs}, \mathrm{TAC}+\mathrm{RAGE}^{-1-}, \mathrm{TAC}+3$-methyladenine (3-MA), and $\mathrm{TAC}+$ chloroquine (CQ). From 4 weeks postoperatively, Sham + AGEs and TAC + AGEs mice were generated by intraperitoneal (i.p.) injection of AGEs $(10 \mathrm{mg} / \mathrm{kg} /$ day $) ; \mathrm{TAC}+3-\mathrm{MA}$ and $\mathrm{TAC}+\mathrm{CQ}$ mice were generated by i.p. injection of 3-MA $(15 \mathrm{mg} / \mathrm{kg} /$ day, dissolved in 5\% DMSO and 95\% saline, Cat. M9281, Sigma, Saint Louis, MO, USA) and CQ $(20 \mathrm{mg} / \mathrm{kg} /$ day, dissolved in 5\% DMSO and 95\% saline, Cat.C6628, Sigma). Sham and TAC groups were administered $0.9 \%$ sodium chloride. All animals were sacrificed for analysis at 8 weeks.

\section{Mouse model of TAC}

Mice were anaesthetized by an i.p. injection of pentobarbital sodium $(50 \mathrm{mg} / \mathrm{kg}$, Sigma). Sham-operated mice underwent a thoracotomy procedure without the constriction of the aorta. In the TAC group, we used a 6-0 silk suture ligature to constrict transverse aorta against a blunted 27-gauge needle to yield a narrowing to $25-30 \%$ of its original cross-sectional area ${ }^{38}$.

\section{Echocardiography}

Echocardiography was performed 8 weeks postoperatively by using a Vevo 2100 Imaging System (VisualSonics Inc., Toronto, ON, Canada) in mice under anesthesia with 1-2.5\% isoflurane (RWD Life Science Co., Guangdong, China) inhalation. The M-mode echocardiogram of the mid-ventricle was examined in shortaxis view. Digital images were analyzed in a blinded manner. Cardiac function indices included left ventricular ejection fraction (LVEF\%), left ventricular fractional shortening (LVFS\%), end-diastolic left ventricular internal dimension (LVIDd), end-systolic left ventricular internal dimension (LVIDs), left ventricular end-diastolic volume (LVEDV), and left ventricular end-systolic volume (LVESV).

\section{Histological examination of cardiac fibrosis}

Heart tissue was harvested and rinsed with cold phosphate-buffered saline (PBS), fixed in $4 \%$ paraformaldehyde over $24 \mathrm{~h}$ at room temperature, then dehydrated and embedded in paraffin. Five- $\mu$ m-thick slices were cut at the papillary muscle level for staining. Masson's trichrome staining was scanned by using Caseviewer 2.0 (Panoramic 250/MIDI, 3D HISTECH, Budapest, Hungary). The Sirius red staining was photographed under polarized light. Quantification of all data was done using Image J (National Institutes of Health, Bethesda, MD, USA) software. At least nine fields per slides of six tissues sections per mouse were analyzed.

\section{Transmission electron microscopy (TEM)}

Left ventricular tissue was harvested and cut into $1 \mathrm{~mm} \times 1 \mathrm{~mm} \times 1 \mathrm{~mm}$ pieces. Samples were fixed in $2.5 \%$ glutaraldehyde over $2 \mathrm{~h}$ at room temperature and transferred to $4{ }^{\circ} \mathrm{C}$. After transferred to $1 \%$ osmium PBS buffer 
for $2 \mathrm{~h}$, the samples were successively placed in ascending series of ethanol $(30,50,70,80,95,100$, and 100\%), $15 \mathrm{~min}$ each. Then permeated overnight with a $1: 1 \mathrm{mix}-$ ture of acetone, followed by soaking in 812 embedding agents at $60^{\circ} \mathrm{C}$ for $48 \mathrm{~h}$. The slices were 60 -nm thick. Uranium-lead double staining was performed, and sections were imaged by TEM (JEOL 1400 Plus TEM system, JEOL Inc., Peabody, MA, USA) at $120 \mathrm{kV}$. The total autolysosomes were quantified according to the average value of nine fields beyond with six tissue sections in each group.

\section{Flow cytometry}

Heart tissue was minced and placed into $60 \%$ collagenase II (A004174, Sangon Biotech, Shanghai, China) in Hanks' solution with $\mathrm{Ca}^{2+}$ and $\mathrm{Mg}^{2+}$ (H1025, Solarbio, Beijing, China), and shaken at $37^{\circ} \mathrm{C}$ for $20 \mathrm{~min}$. Subsequently, the cells were passed through $70-\mu$ m nylon mesh and centrifuged $\left(15 \mathrm{~min}, 300 \times g, 4{ }^{\circ} \mathrm{C}\right)$. Cells were stained with APC-labeled anti-VE-cadherin (vascular endothelial cadherin) (EB-50-1441-82), PE/Cy7-labeled anti-CD31 (EB-25-0311-82), and FITC-labeled anti- $\alpha$-SMA (EB-539760-82) for $20 \mathrm{~min}$ at room temperature. Finally, the stained single cells were resuspended in staining buffer and analyzed using a flow cytometer (MoFloAstrios EQs, BECKMAN, Bria, California, USA).

\section{Cell culture and adenovirus shRNA preparation and application}

HUVECs (Cat.8000) were purchased from ScienCell Research Laboratories (Carlsbad, CA, USA) and cultured in ECM medium (Cat.1001) supplemented with 5\% fetal bovine serum (Cat.0025), 1\% endothelial cell growth supplement (Cat.1052), and 1\% penicillin/streptomycin solution (Cat.0503) in a humidified $5 \% \mathrm{CO}_{2}$ incubator at $37^{\circ} \mathrm{C}$. On day 4, HUVECs were treated with AGEs (Cat.2221-10, BioVision, Milpitas, CA, USA) at 50, 100, 200 , or $400 \mu \mathrm{g} / \mathrm{ml}$ for $24 \mathrm{~h}$ and AGEs at $200 \mu \mathrm{g} / \mathrm{ml}$ for 6 , 12,24 , and $48 \mathrm{~h}$.

Adenoviruses with humanBECN1 (Ad-SiBECN1) were constructed by Genechem (Shanghai, China). Twentyfour hours after transfection, the adenovirus was removed, and transfection efficiency of Ad-SiBECN1 was evaluated by GFP fluorescent protein in the cells.

\section{Immunofluorescence staining}

In vivo, after paraffin sections were dewaxed and dehydrated using xylene and graded ethanol series, antigens were retrieved by sodium citrate heating. Endogenous peroxidase was removed by adding $30 \% \mathrm{H}_{2} \mathrm{O}_{2}$. An immunohistochemistry pen was used to draw a circle around the tissue. The sections were incubated with $0.3 \%$ Triton X-100/PBS at room temperature for $20 \mathrm{~min}$ and incubated with mouse IgG-blocking solution (M.O.M Kit,
Vector Laboratories, Burlingame, CA, USA) diluted in $0.01 \%$ Triton X-100/PBS at room temperature for $1 \mathrm{~h}$, after $5 \%$ goat serum (SL038, Solarbio, Beijing, China) was added to block the tissue for $30 \mathrm{~min}$ at room temperature. Subsequently, the sections were incubated overnight at $4{ }^{\circ} \mathrm{C}$ with the primary anti-CD31 antibody (ab28364, 1:60, Abcam, Cambridge, UK) and anti- $\alpha$-SMA antibody (Abcam, ab7817, 1:200). After washing three times with PBS each for $3 \mathrm{~min}$, secondary fluorescent antibodies were prepared at a ratio of 1:1000. The sections were incubated in the dark for $1 \mathrm{~h}$ at room temperature and then washed with PBS three times for 3 min each. Finally, DAPI solution was added to the sections for 5 min followed by three PBS washes.

In vitro, the harvested cells were fixed in $4 \%$ paraformaldehyde at room temperature, then incubated with $0.1 \%$ Triton X-100/PBS at room temperature for $20 \mathrm{~min}$, and incubated with 5\% goat serum (SL038, Solarbio) for $1 \mathrm{~h}$ at room temperature. The cells were incubated with primary anti-CD31 antibody (Abcam, ab28364, 1:200) and anti- $\alpha$-SMA antibody (Abcam, ab7817, 1:200) at $4{ }^{\circ} \mathrm{C}$ overnight. The incubation method with secondary antibody and DAPI was same as described above for the tissue samples.

A confocal scanning microscope was used to image the slices in a dark room.

\section{Quantitative real-time PCR (qPCR)}

Total RNA was extracted from the left ventricular tissue and cells by RNAzol RT (Molecular Research Center, Cincinnati, OH, USA). First-strand cDNA was synthesized with a FastKing RT Kit (Tiangen, Beijing, China). The cDNA was used to perform quantitative PCR on a CFX96 Real-Time System (Bio-Rad Laboratories, Inc., Hercules, CA, USA) using the SYBR Select master mix kit (Applied Biosystems, Austin, TX, USA). The PCR conditions were $2 \mathrm{~min}$ at $50{ }^{\circ} \mathrm{C}$ and $30 \mathrm{~s}$ at $95^{\circ} \mathrm{C}$ followed by 40 cycles of $95^{\circ} \mathrm{C}$ for $15 \mathrm{~s}, 60^{\circ} \mathrm{C}$ for $1 \mathrm{~min}$, and $15 \mathrm{~s}$ at $65^{\circ} \mathrm{C}$. The specific primers used are listed in Table 1. Relative amounts of mRNA for specific genes were calculated using $2^{-\Delta \Delta C t}$ values. Each sample was run in duplicate, and the mean value of each set of duplicates normalized to that of mouse or human GAPDH was used to calculate relative gene expression.

\section{Western blot}

Left ventricular tissue was harvested and lysed using a Minute $^{\mathrm{TM}}$ Total Protein Extraction Kit for Cultured Cells and Tissues (Cat.SD-001/SN-002, Invent Biotechnologies, Plymouth, MN, USA). After denature proteins fully, lysates were subjected to SDS-PAGE and transferred to $0.45 \mu \mathrm{m}$ polyvinylidene fluoride membranes (Cat.1620260, Bio-Rad Laboratories, Inc.). Membranes were blocked with 5\% skim milk (Cat.9999, Cell Signaling 
Table 1 Primer sequence.

\begin{tabular}{lll}
\hline Gene & Forward sequence & Reverse sequence \\
\hline m-GAPDH & GGCTGTATTCCCCTCCATCG & CCAGTTGGTAACAATGCCATGT \\
m-COL I & TGGCCTTGGAGGAACTTTG & CTTGGAACCTTGTGGACCAG \\
m-COL III & CTGTAACATGGAAACTGGGGAAA & CCATAGCTGAACTGAAAACCACC \\
m-CD31 & ACCGGGTGCTGTTCTATAAGG & TCACCTCGTACTCAATCGTGG \\
m-VE-Cadherin & CACTGCTITGGGAGCTTC & GGGGCAGCGATTCATTITCT \\
m-a-SMA & GTCCCAGACATCAGGGAGTAA & TCGGATACTTCAGCGTCAGGA \\
m-N-Cadherin & AGCGCAGTCTTACCGAAGG & TCGCTGCTTTCATACTGAACTIT \\
H-GAPDH & CCATGGAGAAGGCTGGGG & CAAAGTTGTCATGGATGACC \\
H- CD31 & GCGAGTCATGGCCCGAAGGC & GGTGGTGCTGACATCCGCGA \\
H-a-SMA & CTATGAGGGCTATGCCTTGCC & GCTCAGCCAGTAGTAACGAAGGA \\
H-VE-Cadherin & GTCACCTTCTGCGAGGATATG & GATGGTGAGGATGCAGAGTAAG \\
H-N-Cadherin & TCAGGCGTCTGTAGAGGCTT & ATGCACATCCTTCGATAAGACTG \\
\hline
\end{tabular}

Technology [CST], Danvers, MN, USA) at room temperature for $1 \mathrm{~h}$ and incubated overnight at $4{ }^{\circ} \mathrm{C}$ with 1:1000 GAPDH (CST, 2118L), 1:1000 RAGE (Abcam, 3611), 1:1000 Beclin 1 (CST, 3495S), 1:1000 LC3B (CST, 2775S), 1:500 CD31 (Abcam, 28364), 1:1000 $\alpha-S M A$ (Abcam, 7817), 1:1000 VE-Cadherin (Abcam, 33168), 1:1000 N-Cadherin (Proteintech, Rosemont, IL, USA, 22018-1-AP), and 1:1000 Collagen type I (COL 1) (Proteintech,14695-1-AP). Membranes were then incubated with HRP-conjugated secondary antibody (CST, 7074 or 7076) for $1 \mathrm{~h}$ at room temperature. Finally, the densities of the protein bands were quantified using Immobilon Western Chemiluminescent HRP Substrate (WBKLS0500, Millipore, Bedford, MA, USA) and visualized using a chemiluminescence system (Bio-Rad Laboratories, Inc.).

\section{Collagen gel contraction assay}

The cell contraction assay was performed using the cell contraction assay kit (CBA-201, Cell Biolabs, San Diego, CA, US). Briefly, HUVECs transfected with or without Ad-SiBECN1 and Ad-SiRAGE were harvested and resuspended in medium. Collagen Gel Mix was prepared according to manufacturer protocol on ice. Next, HUVECs were resuspended at a concentration of $2 \times 10^{6}$ cells $/ \mathrm{ml}$. An amount of $200 \mu \mathrm{l}$ cell suspension was mixed with $800 \mu \mathrm{l}$ collagen gel, after vigorous vortex, the total $1 \mathrm{ml}$ cell suspension was added into each well of a 12-well tissue culture plate and incubated at $37^{\circ} \mathrm{C}$ for $1 \mathrm{~h}$. The control group, Ad-SiRAGE, and Ad-siBECN1 group were added to $1 \mathrm{ml}$ culture medium, and others were added to $1 \mathrm{ml}$ culture medium containing $200 \mu \mathrm{g} / \mathrm{ml}$ AGEs. Photographs were taken at 0 and $24 \mathrm{~h}$ to quantitate the gel contraction area ratio using Image J software.

\section{Statistical analysis}

Values are presented as mean \pm standard error of mean. Differences between experimental groups were determined by one-way or two-way ANOVA, followed by post hoc Fisher Tukey's multiple comparison test. Analyses were carried out with Prism 7 (GraphPad, San Diego, CA, USA). $p<0.05$ was considered statistically significant.

\section{Results}

RAGE knockout improves cardiac function at 8 weeks after TAC

To clarify the potential influence of AGEs/RAGE on HF, we examined cardiac function at 8 weeks. The LVEF and LVFS were markedly reduced in the TAC group, while LVEDV, LVESV, LVIDd, and LVIDs were significantly increased, suggesting an impairment (such as organ ischemia, and hypoxia) of cardiac function. There was no apparent difference among Sham groups, or between the TAC and TAC + AGEs group. A striking improvement was seen in $\mathrm{TAC}+\mathrm{RAGE}^{-1-}$ group compared with the TAC group (Fig. 1).

\section{RAGE knockout attenuates cardiac fibrosis through mediating EndMT in HF mice}

On histological examination, the TAC group showed marked fibrosis in the myocardium, especially in the perivascular area, relative to Sham controls. RAGE knockout prevented increased fibrogenesis both in the myocardium and perivascular area (Fig. 2A, B). On exploring perivascular collagen deposition, we found that COL 1 and collagen type III (COL 3 ) accumulated in TAC group, and COL 1 increased significantly over COL 3 . In contrast, there was obvious reduction in both COL 1 and COL 3 in RAGE knockout mice (Fig. 2A, C-E). There was 


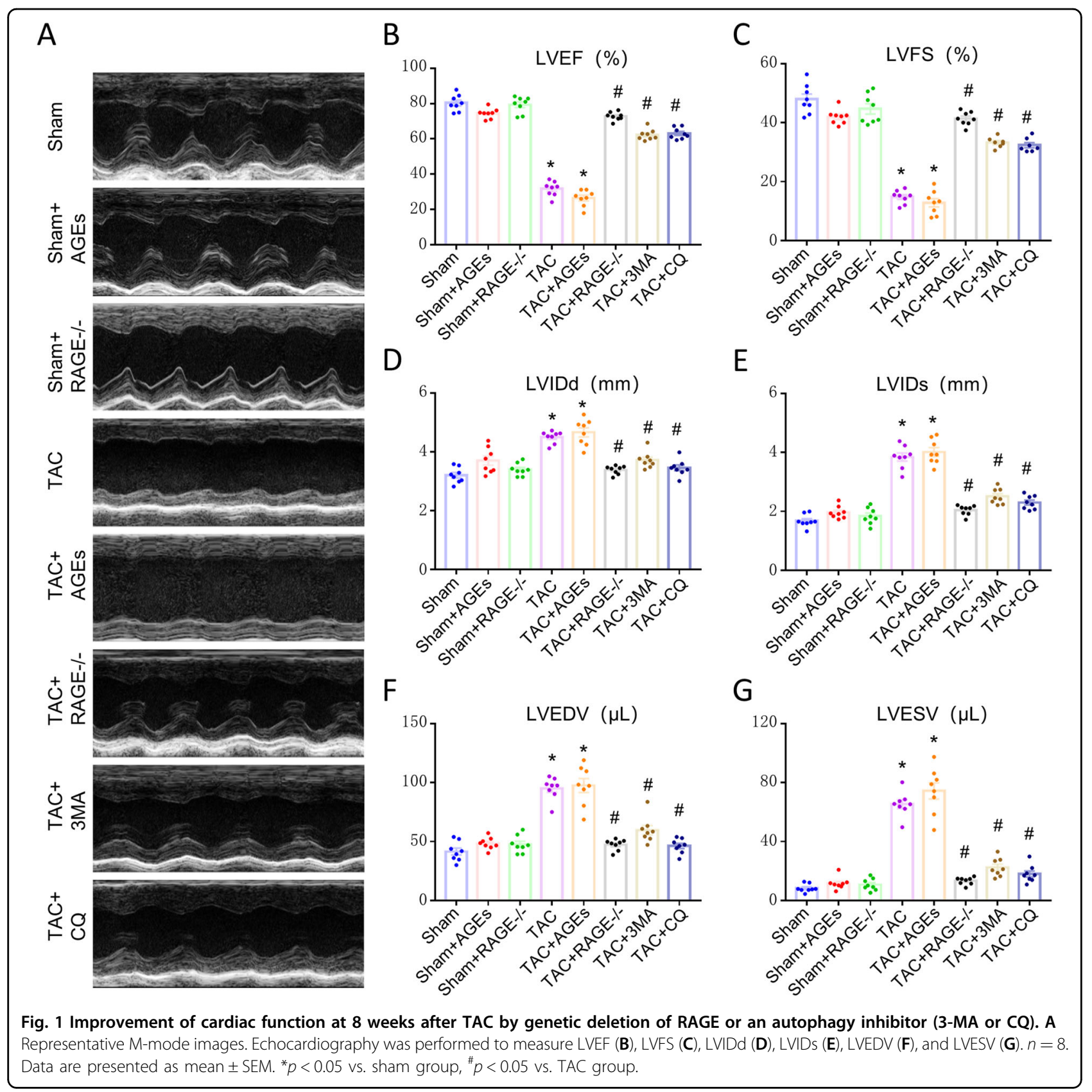

no significant difference among Sham groups or the TAC and TAC + AGEs group.

As the perivascular area was most severely affected by fibrosis, we hypothesized that EndMT contributes to cardiac fibrosis in HF mice. We performed double immunofluorescence staining with antibodies to CD31 and $\alpha$-SMA and found that CD31 was markedly downregulated in myocardial vessels in the TAC group and mice treated with AGEs, while this down-regulation was reversed upon RAGE knockout. In contrast, $\alpha$-SMA was up-regulated in the myocardial vessels of the TAC group, TAC + AGEs group and Sham + AGEs group; however, this up-regulation was also reversed in RAGE knockout mice (Fig. 3A-C). Expression of CD31 and VE-Cadherin (markers of endothelial cells) were decreased, whereas expression of $\alpha$-SMA and $\mathrm{N}$-Cadherin (markers of mesenchymal cells) were increased in HF mice and mice treated with AGEs (Fig. 3D-G). Again, RAGE knockout reversed these mRNA changes.

Flow cytometry was used to study whether EndMT is one origin of myofibroblasts in HF. Compared with the Sham control, there was a 2.69-fold decrease in the number of $\mathrm{CD}_{3} 1^{+} / \mathrm{VE}-\mathrm{Cadherin}{ }^{+}$endothelial cells, and about a 2.85 -fold increase in the number of $\mathrm{CD}^{+} 1^{+} / \alpha$ - 


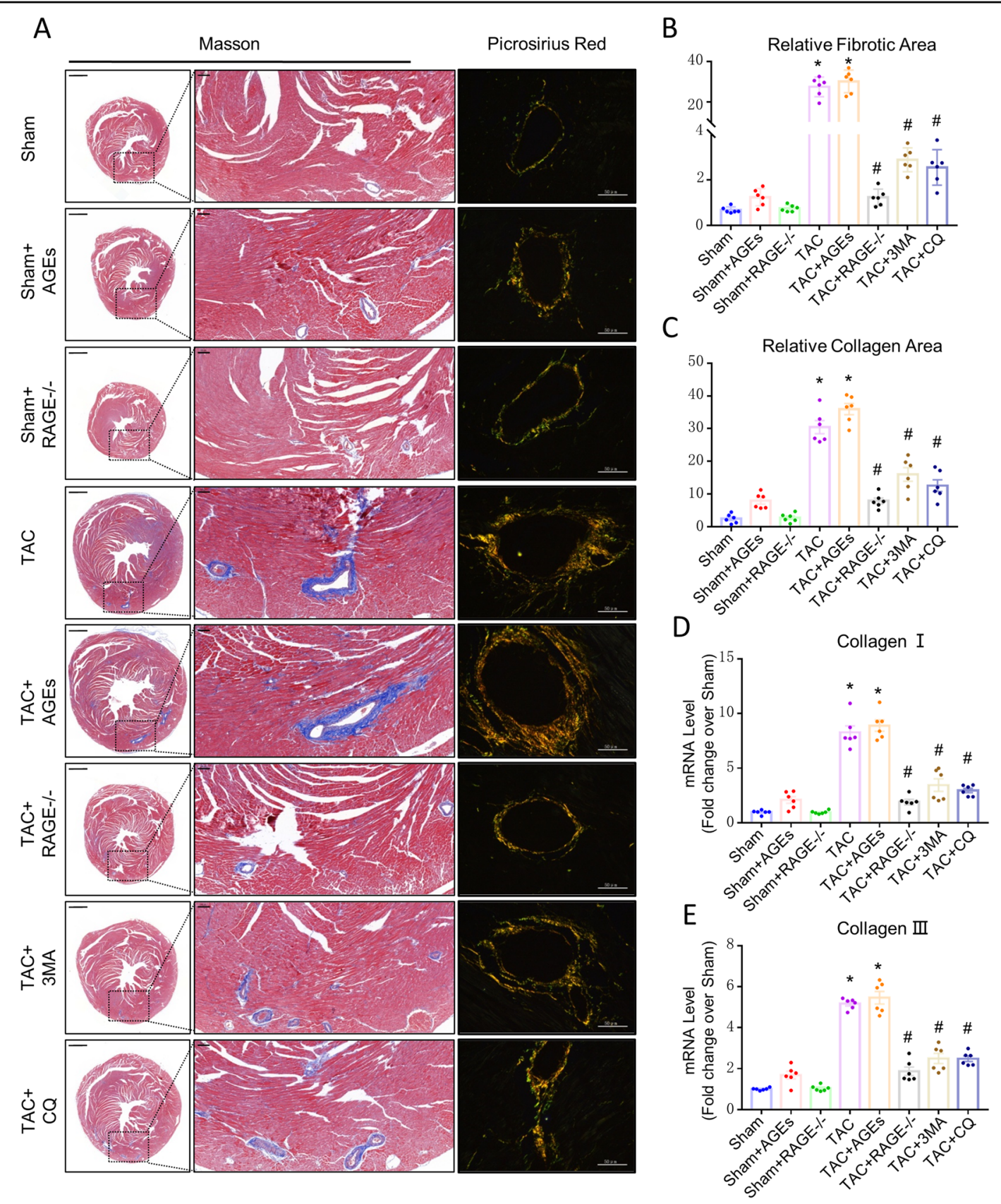

Fig. 2 Effect of AGEs/RAGE or autophagy on cardiac fibrosis after TAC. A Myocardial fibrosis was detected by Masson's trichrome staining (scale bar: transverse sections $=1000 \mu \mathrm{m}$, perivascular sections $=200 \mu \mathrm{m}$ ), and perivascular collagen synthesis was detected by a Sirius red-polarized method (scale bar $=50 \mu \mathrm{m}$ ). Blue areas indicate fibrosis. Yellow areas indicate collagen I, and green areas indicate collagen III. The fibrosis area was measured with a quantitative digital image analysis system: fibrotic area (B) and collagen deposition area (C). mRNAs for fibrosis associated genes collagen I (D) and collagen III (E) were measured by $q$ PCR. $n=6$. Data are presented as mean \pm SEM. ${ }^{*} p<0.05$ vs. sham group, ${ }^{*} p<0.05$ vs. TAC group.

$\mathrm{SMA}^{+}(\alpha$-smooth muscle actin, myofibroblast marker) cells in the TAC group. Only $8.24 \%$ cells co-expressed CD31 and $\alpha$-SMA, while about $22.34 \%$ cells co-expressed CD31 and VE-Cadherin in the Sham group. Moreover, AGEs/RAGE regulated the EndMT, as shown in Fig. 4. Compared with the Sham control, there was a 1.62-fold decrease in the number of CD31 ${ }^{+} / \mathrm{VE}-\mathrm{Cadherin}^{+}$endothelial cells and about a 2.37-fold increase in the number of $\mathrm{CD} 31^{+} / \alpha-\mathrm{SMA}^{+}$cells in the Sham + AGEs group, while compared with the TAC group, there was a 1.94fold increase in the number of CD31 ${ }^{+} / \mathrm{VE}$-Cadherin ${ }^{+}$ endothelial cells, and about a 2.78 -fold decrease in the 


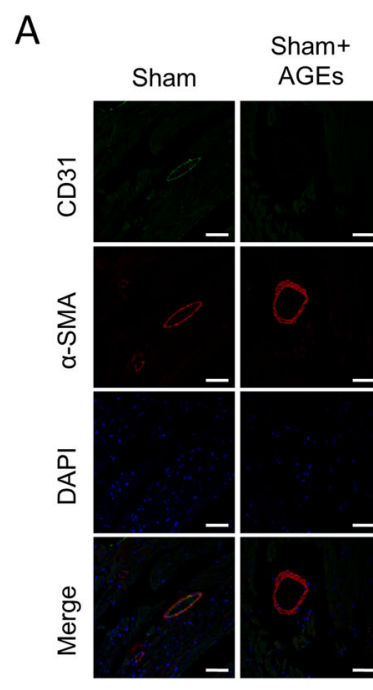

B
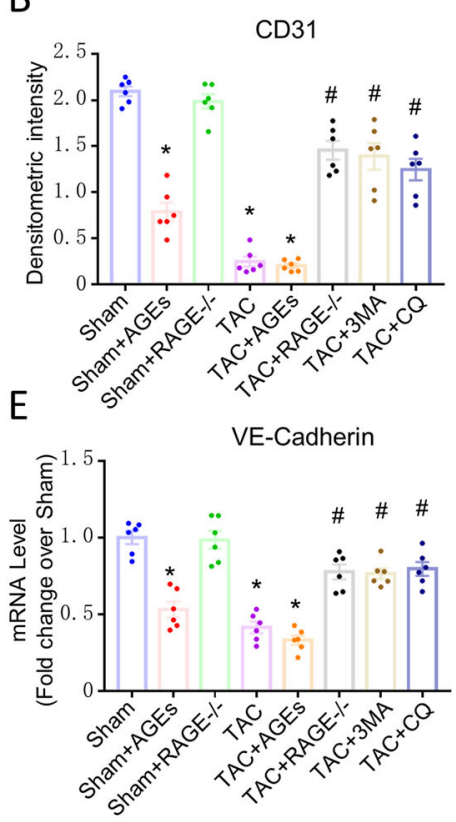

Sham+
RAGE-/-

TAC

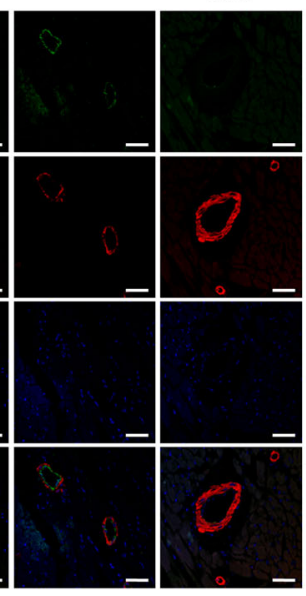

C
TAC+
AGEs
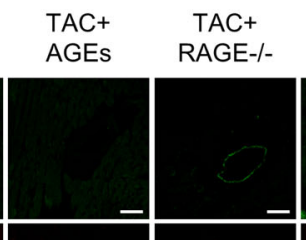

TAC+

TAC+

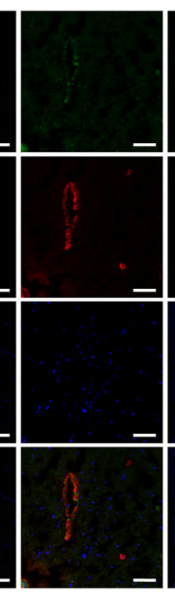

D
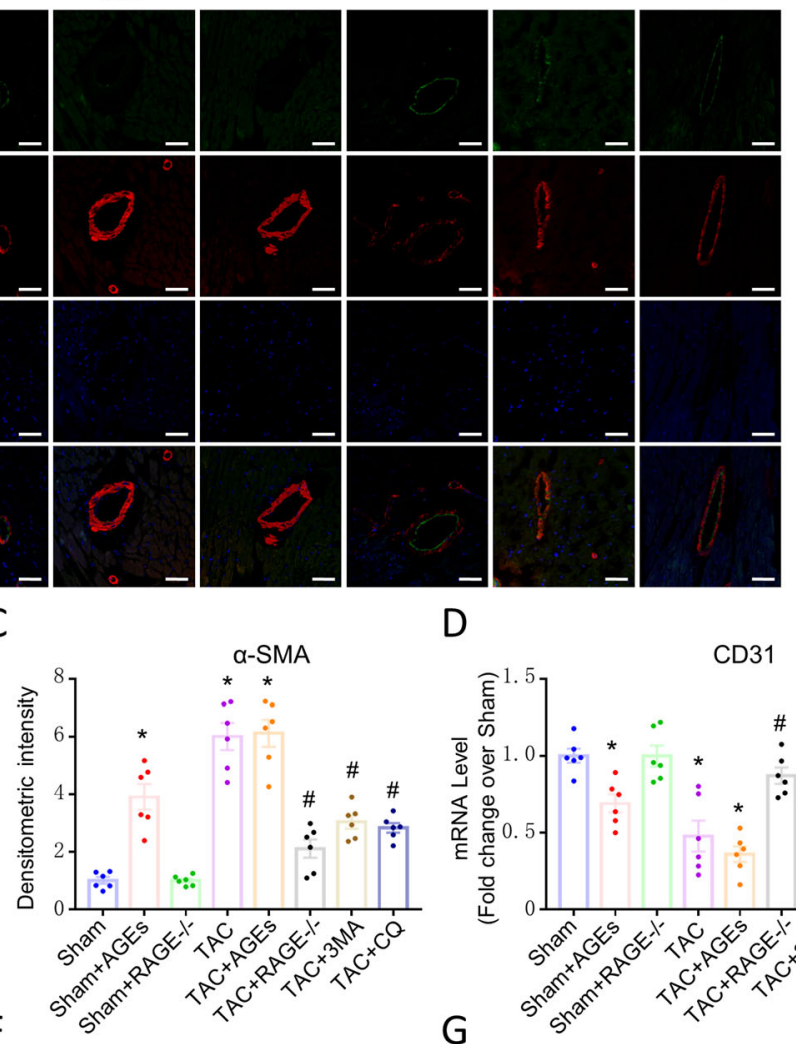

G
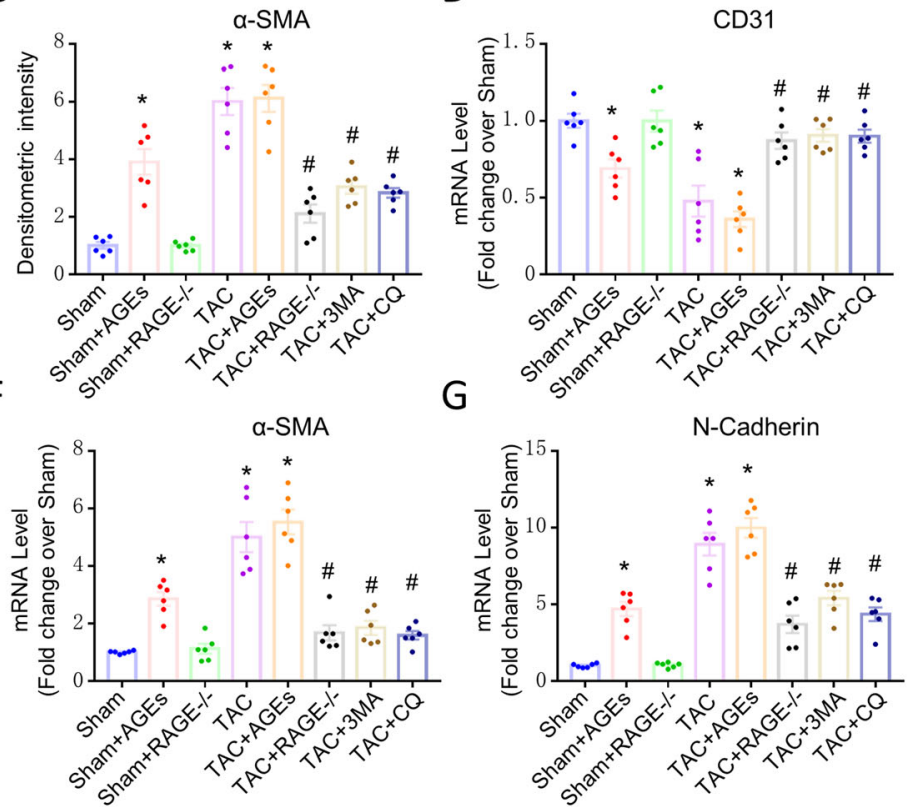

Fig. 3 Endothelial cells may transition to mesenchymal cells in HF. A Confocal microscopic image of double immunofluorescence staining with CD31 (green) and a-SMA (red); nuclei were counter stained with DAPI (blue); Scale bars $=100 \mu \mathrm{m}$. B, C Quantification of immunofluorescence analysis. mRNAs for endothelial cells-associated genes [CD31 (D), VE-Cadherin (E)] and mesenchymal cells-associated gene [a-SMA (F) and NCadherin $(\mathbf{G})$ ] were measured by qPCR. $n=6$. Data are presented as mean \pm SEM. ${ }^{*} p<0.05$ vs. sham group, ${ }^{\#} p<0.05$ vs. TAC group.

number of $\mathrm{CD} 31^{+} / \alpha-\mathrm{SMA}^{+}$cells in the TAC $+\mathrm{RAGE}^{-1-}$ group, but no difference in the TAC + AGEs group.

\section{AGEs/RAGE could regulate autophagy}

Autolysosomes of cardiac tissues were analyzed by TEM. Increased typical autolysosomes were detected in the TAC group, TAC + AGEs group, and Sham + AGEs group, while Sham controls showed normal myocardial fine structure. Like the autophagy inhibitors, RAGE knockout inhibited autophagic activity (Fig. 5A, B). Western blot showed increased expression of proteins related to autophagy (LC3BII/I and Beclin 1) in the TAC group, TAC + AGEs group, and Sham + AGEs group. However, LC3BII/I and Beclin 1 protein expression levels were significantly lower in the TAC $+\mathrm{RAGE}^{-1-}$ group compared with the TAC group (Fig. 5C-F).

\section{Autophagy inhibitors alleviate cardiac fibrosis through mediating EndMT}

To examine whether autophagy could regulate EndMT, we treated mice with 3-MA (TAC + 3-MA) and CQ (TAC $+\mathrm{CQ}$ ). Echocardiography results (Fig. 1) and histological 

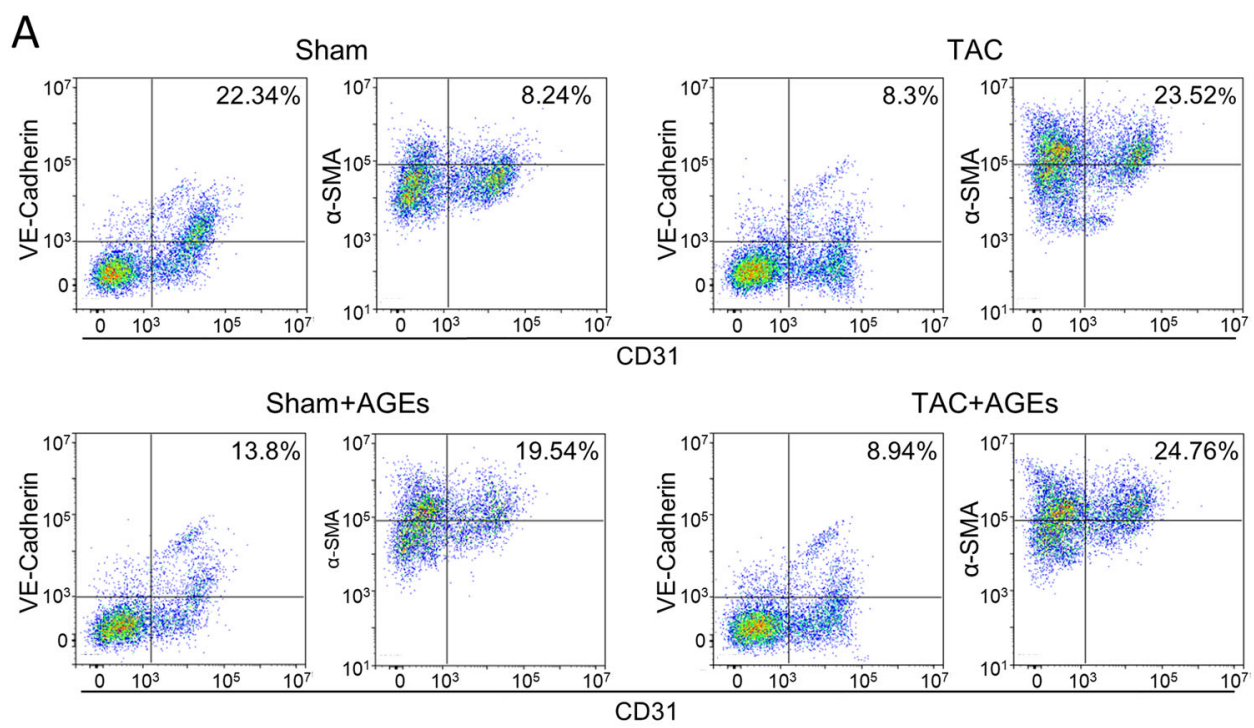

Sham+RAGE-/-

TAC+RAGE-/-
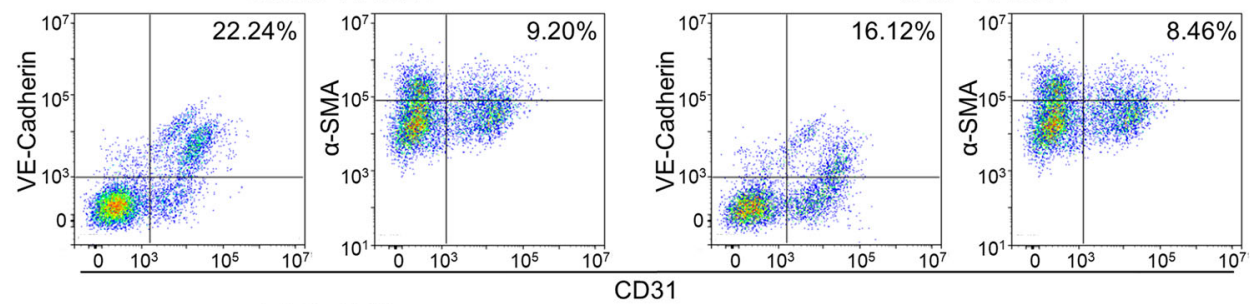

TAC+3MA

$\mathrm{TAC}+\mathrm{CQ}$
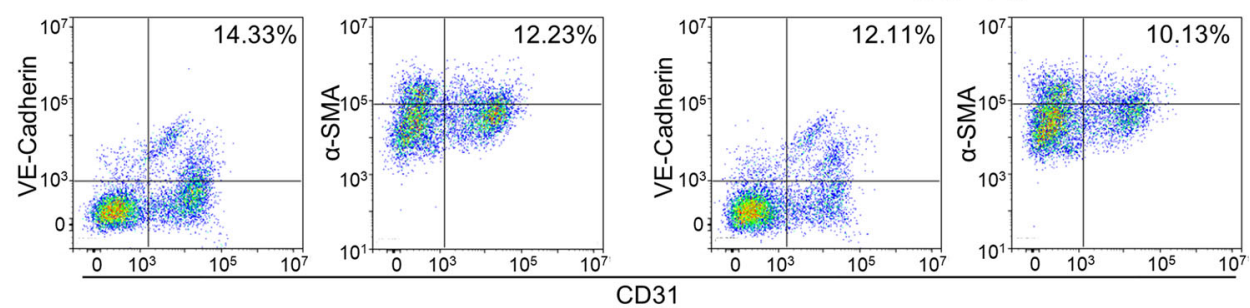

B

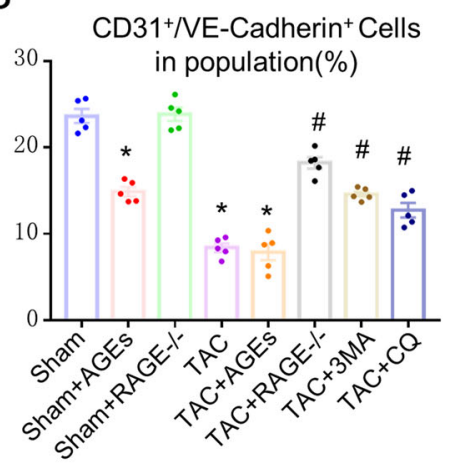

C

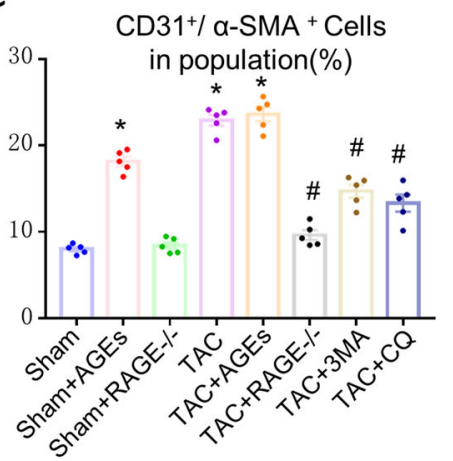

Fig. 4 EndMT is one origin of myofibroblasts in HF and could be regulated by AGEs/RAGE and autophagy. A Flow cytometry analysis of primary isolates of mouse left ventricular cells. After mechanical disaggregation and enzymatic digestion, single-cell suspensions were incubated with combination of fluorescent antibodies to CD31, VE-Cadherin, and a-SMA. B, C Quantification of co-expressed cell analysis. $n=5$. Data are presented as mean \pm SEM. ${ }^{*} p<0.05$ vs. sham group, ${ }^{\#} p<0.05$ vs. TAC group. 

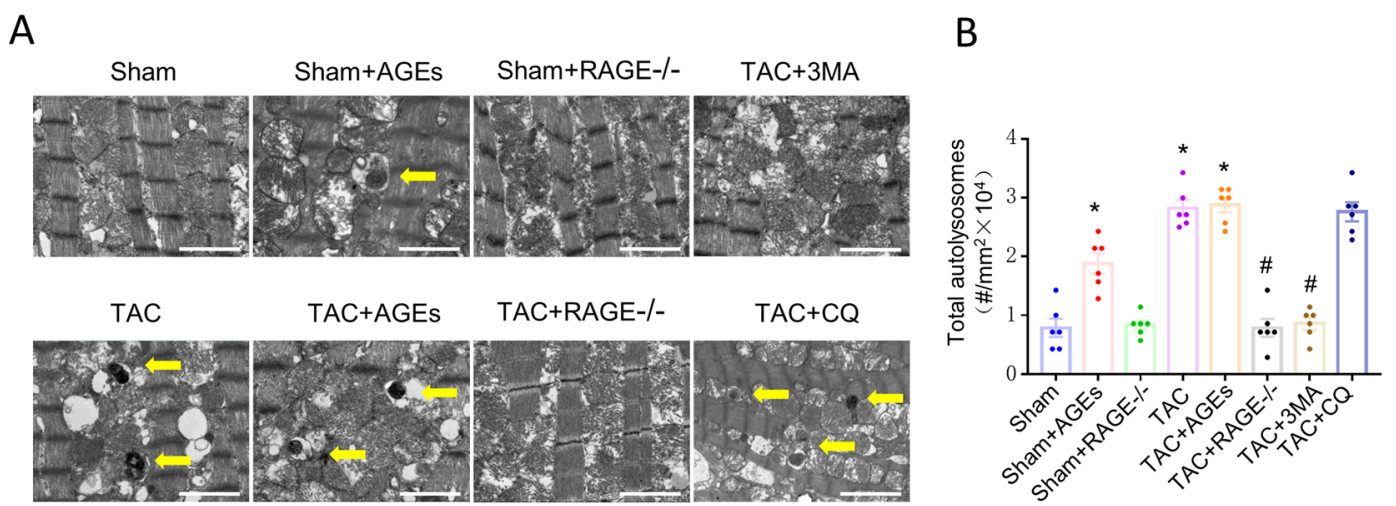

C

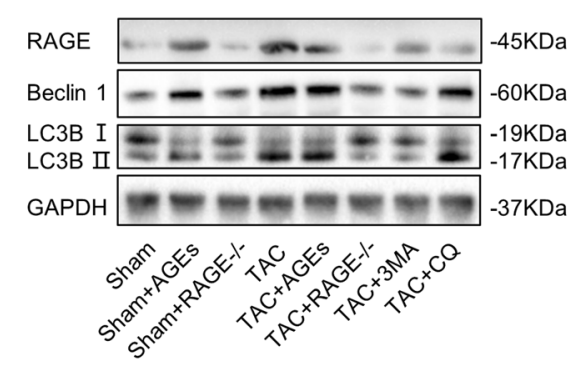

E

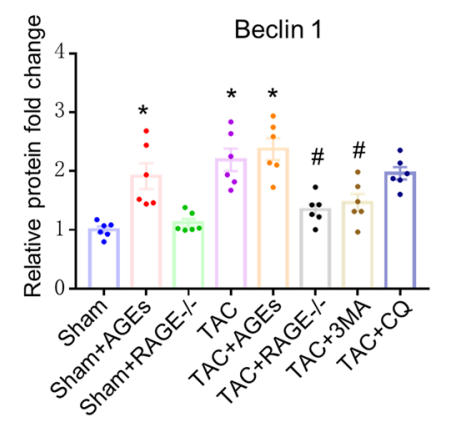

D

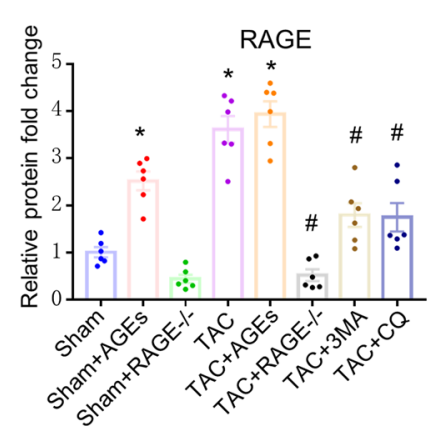

$\mathrm{F}$

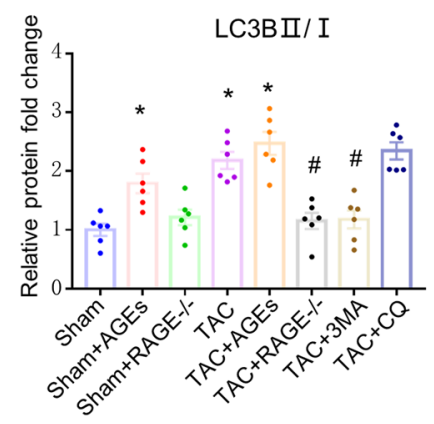

Fig. 5 Reduction of autophagy at 8 weeks after TAC by genetic deletion of RAGE or an autophagy inhibitor (3-MA or CQ). A Representative autophagic ultrastructure of the heart tissue under transmission electron microscopy (Scale bars $=2 \mu \mathrm{m}$; arrow: autolysosome). B Quantification of autophagy lysosomes analysis. C-F Left ventricular levels of RAGE and autophagy-related proteins (LC3BII/I and Beclin 1) were assessed by western blot. Quantification of RAGE (D), Beclin 1 (E), and LC3BII/I (F). GAPDH was used as the internal control. $n=6$. Data are presented as mean \pm SEM. ${ }^{*} p<$ 0.05 vs. sham group, ${ }^{\#} p<0.05$ vs. TAC group.

examination (Fig. 2) suggested that inhibition of excessive autophagy ameliorated cardiac fibrosis and cardiac function in HF. 3-MA and CQ mice both prevented the downexpression of CD31 and VE-Cadherin, the up-expression of $\alpha$-SMA and N-Cadherin at mRNA level (Fig. 3), as well as the decrease in CD31 $1^{+} / \mathrm{VE}-\mathrm{Cadherin}^{+}$endothelial cells and increase in $\mathrm{CD}^{+} 1^{+} / \alpha$-SMA ${ }^{+}$cells (Fig. 4), implying that autophagy may mediate EndMT in HF.

\section{AGEs/RAGE mediated EndMT by regulating autophagy activity}

To clarify the regulatory mechanisms of EndMT, we cultured HUVECs and performed Ad-siRNA transfection to knockout BECN1 expression in the cultured cells. Treatment with $200 \mu \mathrm{g} / \mathrm{ml}$ AGEs for $24 \mathrm{~h}$ was optimal to induce EndMT of HUVECs, reflected in loss of cell-cell junctions, cobblestone morphology, and translation into long filopodia characteristic of mesenchymal cells. The expression of mesenchymal cells were significantly upregulated and endothelial cells were down-regulated, according to the mRNA level (Supplementary Fig. 1) and the protein expression level (Fig. 6B, D-H). Interestingly, Beclin 1 knockout prevented EndMT in HUVECs subjected to AGEs (Fig. 6). Then, we detected the cell contractility using a three-dimensional collagen lattice and found that AGEs treatment significantly increased cell 


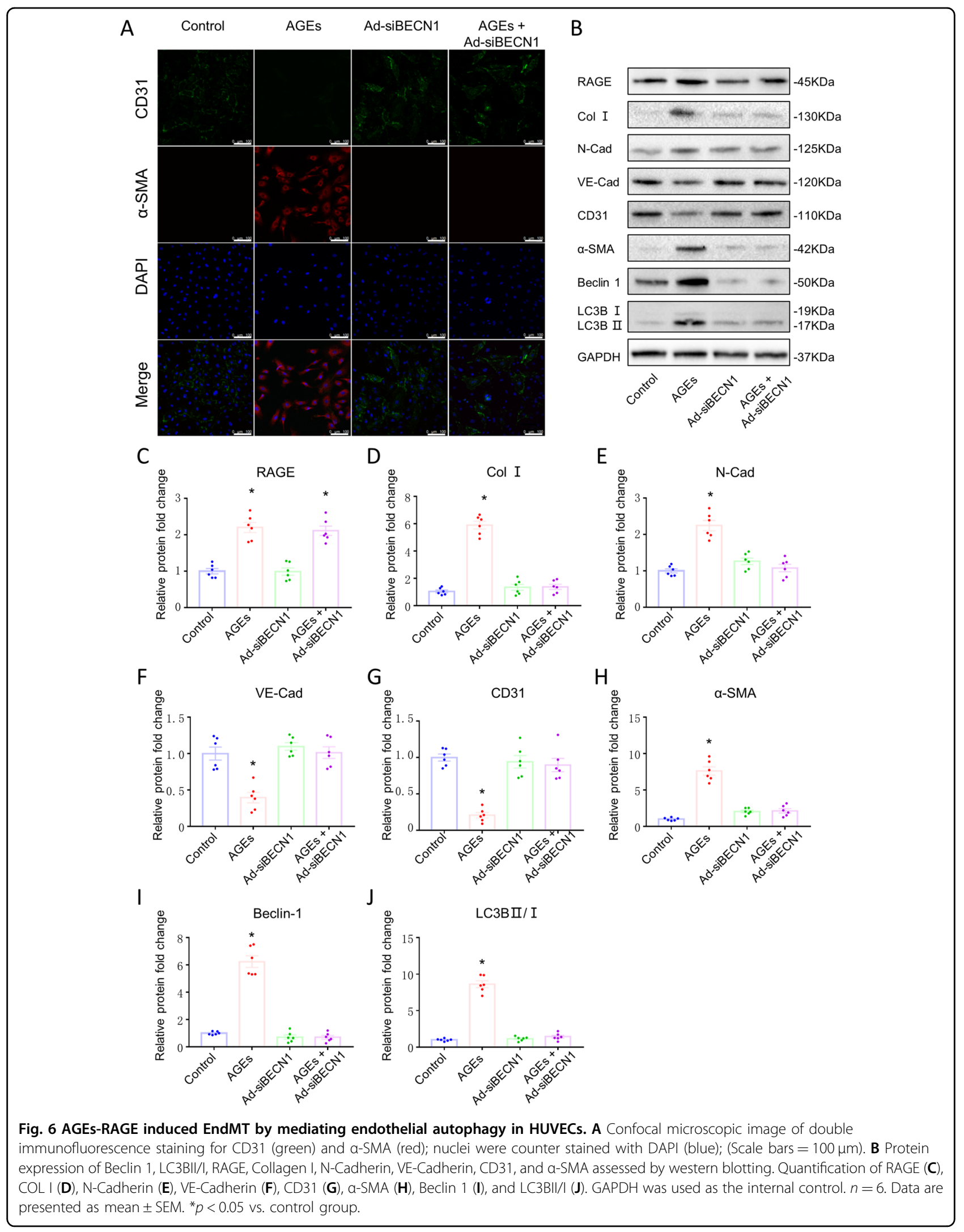


contractility after $24 \mathrm{~h}$, with gel size reduced to $50 \%$ compared to controls. Beclin 1 knockout and RAGE knockout significantly repressed the cell contraction induced by AGEs (Supplementary Fig. 2). These findings suggest that AGEs/RAGE may mediate EndMT by regulating autophagy activity.

\section{Discussion}

The present study investigated the regulatory mechanisms of EndMT in cardiac fibrosis of overload-induced HF. We found that EndMT may be the source of myofibroblasts and showed that AGEs could induce EndMT and autophagy. In addition, our data showed RAGE knockout could effectively inhibit autophagy, reduce EndMT, ameliorate myocardial fibrosis and cardiac function in HF mice. Autophagy may also be a crucial mediator between AGEs/RAGE and EndMT. Autophagy inhibitors attenuated EndMT as well as cardiac fibrosis and cardiac function. Beclin 1 knockout could directly eradicate AGEs/RAGE-induced EndMT in HUVECs, thus providing useful insights into the AGEs/RAGE-autophagy-EndMT axis as a promising target for the treatment of HF.

Recently, AGEs/RAGE has been regarded as an effective clinical prognostic index of cardiovascular disease, with AGEs level positively correlated with postinfarction HF development ${ }^{39}$. High sRAGE has been associated with deteriorating left ventricular function and an increased rate of HF post-discharge hospitalization ${ }^{40-42}$. Our study showed that RAGE knockout could prevent cardiac fibrosis and COL 1 deposition in TAC-induced HF mice. It has been reported that endomyocardial fibrosis is associated with selective deposition of COL $1^{43,44}$, which is consistent with our results.

Importantly, we found that RAGE knockout could down-regulate expression of $\alpha$-SMA and $\mathrm{N}$-Cadherin, and up-regulate expression of CD31 and VE-Cadherin. VECadherin is a component of endothelial cell-to-cell adherens junction ${ }^{45}$, whereas $\mathrm{N}$-Cadherin increases cell separation and cell motility. The switch from VECadherin to $\mathrm{N}$-Cadherin suggested the occurrence of EndMT in TAC-induced HF mice ${ }^{46}$. We also observed increased co-expression of CD31 and $\alpha$-SMA in cardiac cells. $\alpha$-SMA is characteristic of activated myofibroblasts involved in pathological cardiac remodeling and subsequent cardiac fibrosis in $\mathrm{HF}^{3}$. Accordingly, our results suggest that RAGE knockout could inhibit EndMT and EndMT may be one of the vital sources of myofibroblasts involved in cardiac fibrosis in HF. Previous studies have identified AGEs as triggers of EndMT ${ }^{35,47-49}$. Our results suggested that AGEs could induce EndMT in vivo and in vitro. However, EndMT induced by AGEs did not cause cardiac fibrosis and influence cardiac function in Sham mice treated with AGEs, which may be a compensatory effect under physiological conditions, while RAGE knockout effectively improve cardiac function, reduce EndMT and prevent myocardial fibrosis in HF mice. Therefore, AGEs/RAGE mediated EndMT may be a potential target for treating cardiac fibrosis in HF.

Consistent with our previous study ${ }^{30}$, we identified AGEs/RAGE as a positive regulator of autophagy. Although basal autophagy is important in cells ${ }^{50}$, excessive autophagy is harmful and has a deleterious effect in various diseases ${ }^{51-53}$. Autophagy has become a cardiovascular therapeutic target ${ }^{54,55}$, with growing evidence that inhibition early autophagosome formation could decrease autophagy, resulting in diminishing pathological remodeling induced by severe pressure stress ${ }^{56,57}$. Our study confirmed that 3-MA (the inhibitor of autophagosome formation) and CQ (the blocker of autolysosome degradation) both protected against cardiac fibrosis and reduced EndMT in HF mice at 8 weeks. These findings suggest that excessive autophagic flux may result in cardiac fibrosis via EndMT.

Importantly, our study identified that AGEs/RAGE mediated EndMT by regulating autophagy activity. Autophagic cell death has been shown in HUVECs treated after $24 \mathrm{~h}$ with glycated collagen, a significant component of $\mathrm{AGEs}^{58}$. Our study detected obvious expression of mesenchymal cells, collagen deposition, decreased expression of endothelial markers, and enhanced cellular contractility after AGEs treatment for $24 \mathrm{~h}$. However, the transition was inhibited when autophagy was blocked, suggesting that AGEs/RAGE contribute to EndMTinduced cardiac fibrosis through regulating autophagy in HF.

In summary, autophagy mediated by AGEs/RAGE triggers EndMT in the progression of HF. RAGE knockout could inhibit autophagy, thus resulting in prevention of EndMT, as well as attenuating cardiac fibrosis and improving cardiac function. AGEs/RAGE-autophagyEndMT axis could be an attractive target for $\mathrm{HF}$ treatment.

\section{Acknowledgements}

We would like to thank Kanazawa University, Japan for their RAGE knockout mice, and Dr. Wanling Xuan for helping with the English language edit.

\section{Author details}

'The First Affiliated Hospital, Guangzhou University of Chinese Medicine, Guangzhou 510405, China. ${ }^{2}$ The First Clinical Medical School, Guangzhou University of Chinese Medicine, Guangzhou 510405, China. ${ }^{3}$ Lingnan Medical Research Center, Guangzhou University of Chinese Medicine, Guangzhou 510405, China. ${ }^{4}$ Guangzhou Key Laboratory of Chinese Medicine for Prevention and Treatment of Chronic Heart Failure, Guangzhou 510405, China. ${ }^{5}$ National Clinical Research Base of Traditional Chinese Medicine, Guangzhou 510405, China

\section{Author contributions}

L.W. conceived the study concept, designed most of the experiments, and conducted most of the analyses. L.Z. designed and performed most of the experiments, conducted most of the analyses, and wrote the manuscript. J.H., 
J.W., J.L., Z.C., H.L., B.D., L.W., H.W., B.L., Y.H., and L.L. performed experiments and provided the corresponding analyses. Z.Y. and S.X. conceived the project and contributed to revise manuscript. All authors reviewed the manuscript.

\section{Funding}

This work was supported by grants from the National Natural Science Foundation of China (Nos. 81673920, 81973776, 81973777) and the Excellent Doctoral Dissertation Incubation Grant of First Clinical School of Guangzhou University of Chinese Medicine (No. YB202002).

\section{Conflict of interest}

The authors declare no competing interests.

\section{Ethical approval}

This study was approved by the Animal Care Committee of Guangzhou University of Chinese Medicine and were strictly in accordance with the National Institutes of Health Guidelines for Care and Use of Laboratory Animals (20181212001).

\section{Publisher's note}

Springer Nature remains neutral with regard to jurisdictional claims in published maps and institutional affiliations.

Supplementary information The online version contains supplementary material available at https://doi.org/10.1038/s41419-021-03750-4.

Received: 21 October 2020 Revised: 19 April 2021 Accepted: 22 April 2021 Published online: 11 May 2021

\section{References}

1. Wu, Q. Q. et al. Mechanisms contributing to cardiac remodelling. Clin. Sci. 131, 2319-2345 (2017).

2. van Putten, S., Shafieyan, Y. \& Hinz, B. Mechanical control of cardiac myofibroblasts. J. Mol. Cell. Cardiol. 93, 133-142 (2016).

3. Travers, J. G., Kamal, F. A., Robbins, J., Yutzey, K. E. \& Blaxall, B. C. Cardiac fibrosis: the fibroblast awakens. Circ. Res. 118, 1021-1040 (2016).

4. Delaunay M., Osman H., Kaiser S. \& Diviani D. The role of cyclic AMP signaling in cardiac fibrosis. Cells $\mathbf{9}, 69$ (2020).

5. MacLean, J. \& Pasumarthi, K. B. Signaling mechanisms regulating fibroblast activation, phenoconversion and fibrosis in the heart. Indian J. Biochem. Biophys. 51, 476-482 (2014).

6. Cao, Y., Feng, B., Chen, S., Chu, Y. \& Chakrabarti, S. Mechanisms of endothelial to mesenchymal transition in the retina in diabetes. invest. Ophth. Vis. Sci. $\mathbf{5 5}$ 7321-7331 (2014)

7. Kizu, A., Medici, D. \& Kalluri, R. Endothelial-mesenchymal transition as a novel mechanism for generating myofibroblasts during diabetic nephropathy. Am. J. Pathol. 175, 1371-1373 (2009).

8. Murdoch, C. E. et al. Endothelial NADPH oxidase-2 promotes interstitial cardiac fibrosis and diastolic dysfunction through proinflammatory effects and endothelial-mesenchymal transition. J. Am. Coll. Cardiol. 63, 2734-2741 (2014).

9. Li, Q. et al. Inhibition of miR-21 alleviated cardiac perivascular fibrosis via repressing EndMT in T1DM. J. Cell. Mol. Med. 24, 910-920 (2020).

10. Bischoff, J. Endothelial-to-mesenchymal transition. Circ. Res. 124, 1163-1165 (2019).

11. Piera-Velazquez, S. \& Jimenez, S. A. Endothelial to mesenchymal transition: role in physiology and in the pathogenesis of human diseases. Physiol. Rev. 99, 1281-1324 (2019)

12. Bostrom, K. I., Yao, J., Guihard, P. J., Blazquez-Medela, A. M. \& Yao, Y. Endothelialmesenchymal transition in atherosclerotic lesion calcification. Atherosclerosis 253, 124-127 (2016)

13. Ranchoux, B. et al. Endothelial-to-mesenchymal transition in pulmonary hypertension. Circulation 131, 1006-1018 (2015).

14. Kovacic, J. C., Mercader, N., Torres, M., Boehm, M. \& Fuster, V. Epithelial-tomesenchymal and endothelial-to-mesenchymal transition: from cardiovascular development to disease. Circulation 125, 1795-1808 (2012).

15. Kovacic, J. C. et al. Endothelial to mesenchymal transition in cardiovascular disease: JACC state-of-the-art review. J. Am. Coll. Cardiol. 73, 190-209 (2019).
16. Zeisberg, E. M. et al. Endothelial-to-mesenchymal transition contributes to cardiac fibrosis. Nat. Med. 13, 952-961 (2007).

17. $\mathrm{Xu}, \mathrm{X}$. et al. Epigenetic balance of aberrant Rasal1 promoter methylation and hydroxymethylation regulates cardiac fibrosis. Cardiovasc. Res. 105, 279-291 (2015).

18. Liu, Z. H. et al. SIRT1 activation attenuates cardiac fibrosis by endothelial-tomesenchymal transition. Biomed. Pharmacother. 118, 109227 (2019).

19. Patschan, D., Schwarze, K., Henze, E., Patschan, S. \& Muller, G. A. Endothelial autophagy and endothelial-to-mesenchymal transition (EndoMT) in eEPC treatment of ischemic AKI. J. Nephrol. 29, 637-644 (2016).

20. Marchi, S. et al. Defective autophagy is a key feature of cerebral cavernous malformations. EMBO Mol. Med. 7, 1403-1417 (2015).

21. Zou, J. et al. Autophagy attenuates endothelial-to-mesenchymal transition by promoting Snail degradation in human cardiac microvascular endothelial cells. Biosci. Rep. 37, 1049 (2017)

22. Folkerts, H., Hilgendorf, S., Vellenga, E., Bremer, E. \& Wiersma, V. R. The multifaceted role of autophagy in cancer and the microenvironment. Med. Res. Rev. 39, 517-560 (2019).

23. Shirakabe, A., Ikeda, Y., Sciarretta, S., Zablocki, D. K. \& Sadoshima, J. Aging and autophagy in the heart. Circ. Res. 118, 1563-1576 (2016).

24. Mizushima, N., Levine, B., Cuervo, A. M. \& Klionsky, D. J. Autophagy fights disease through cellular self-digestion. Nature 451, 1069-1075 (2008).

25. Yang, Z. \& Klionsky, D. J. Eaten alive: a history of macroautophagy. Nat. Cell Biol. 12, 814-822 (2010).

26. Nakai, A. et al. The role of autophagy in cardiomyocytes in the basal state and in response to hemodynamic stress. Nat. Med. 13, 619-624 (2007).

27. Matsui, Y. et al. Distinct roles of autophagy in the heart during ischemia and reperfusion: roles of AMP-activated protein kinase and Beclin 1 in mediating autophagy. Circ. Res. 100, 914-922 (2007).

28. Xie, Y. et al. Protective role of autophagy in AGE-induced early injury of human vascular endothelial cells. Mol. Med. Rep. 4, 459-464 (2011).

29. Zhu, H. et al. Cardiac autophagy is a maladaptive response to hemodynamic stress. J. Clin. Invest. 117, 1782-1793 (2007).

30. Gao, W. et al. Inhibiting receptor of advanced glycation end products attenuates pressure overload-induced cardiac dysfunction by preventing excessive autophagy. Front. Physiol. 9, 1333 (2018).

31. Hou, X. et al. Advanced glycation endproducts trigger autophagy in cadiomyocyte via RAGE/PI3K/AKT/mTOR pathway. Cardiovasc. Diabetol. 13, 78 (2014).

32. Huang, C. Y. et al. HMGB1 promotes ERK-mediated mitochondrial Drp1 phosphorylation for chemoresistance through RAGE in colorectal cancer. Cell Death Dis. 9, 1004 (2018).

33. Meng, H. Z., Zhang, W. L., Liu, F. \& Yang, M. W. Advanced glycation end products affect osteoblast proliferation and function by modulating autophagy via the receptor of advanced glycation end products/Raf Protein/Mitogen-activated Protein Kinase/Extracellular Signal-regulated Kinase Kinase/ Extracellular Signal-regulated Kinase (RAGE/Raf/MEK/ERK) pathway. J. Biol. Chem. 290, 28189-28199 (2015).

34. Paradela-Dobarro, B. et al. Evolution and bad prognostic value of advanced glycation end products after acute heart failure: relation with body composition. Cardiovasc. Diabetol. 16, 115 (2017).

35. Russo, I. \& Frangogiannis, N. G. Diabetes-associated cardiac fibrosis: cellular effectors, molecular mechanisms and therapeutic opportunities. J. Mol. Cell. Cardiol. 90, 84-93 (2016).

36. He, W. Zhang, J., Gan, T. Y., Xu, G. J. \& Tang, B. P. Advanced glycation end products induce endothelial-to-mesenchymal transition via downregulating Sirt 1 and upregulating TGF-beta in human endothelial cells. Biomed. Res. Int. 2015, 684242 (2015).

37. Myint, K. M. et al. RAGE control of diabetic nephropathy in a mouse model: effects of RAGE gene disruption and administration of low-molecular weight heparin. Diabetes 55, 2510-2522 (2006).

38. Wang, J. et al. Pyroptosis and ferroptosis induced by mixed lineage kinase 3 (MLK3) signaling in cardiomyocytes are essential for myocardial fibrosis in response to pressure overload. Cell Death Dis. 11, 574 (2020).

39. Raposeiras-Roubin, S. et al. Predictive value of advanced glycation end products for the development of post-infarction heart failure: a preliminary report. Cardiovasc. Diabetol. 11, 102 (2012).

40. Grauen, L. H. et al. The soluble receptor for advanced glycation endproducts (SRAGE) has a dual phase-dependent association with residual cardiovascular risk after an acute coronary event. Atherosclerosis 287, 16-23 (2019). 
41. Qiu, H. et al. Dynamic fluctuations of advanced glycation end products and its C-terminal truncated receptor level in patients with acute ST-segment elevation myocardial infarction and undergoing diabetes or not: a retrospective study. Medicine (Baltimore) 97, e11278 (2018).

42. Paradela-Dobarro, B. et al. The different roles for the advanced glycation end products axis in heart failure and acute coronary syndrome settings. Nutr. Metab. Cardiovasc. Dis. 29, 1050-1060 (2019).

43. Radhakumary, C., Kumari, T. V. \& Kartha, C. C. Endomyocardial fibrosis is associated with selective deposition of type I collagen. Indian Heart J. 53, 486-489 (2001)

44. Chen, Z. et al. A simplified herbal formula for the treatment of heart failure: Efficacy, bioactive ingredients, and mechanisms. Pharmacol. Res. 147, 104251 (2019).

45. Giannotta, M., Trani, M. \& Dejana, E. VE-cadherin and endothelial adherens junctions: active guardians of vascular integrity. Dev. Cell 26, 441-454 (2013).

46. Loh, C. Y. et al. The E-Cadherin and N-Cadherin switch in epithelial-tomesenchymal transition: signaling, therapeutic implications, and challenges. Cells 8, 1118 (2019).

47. Raghavan, C. T. et al. AGEs in human lens capsule promote the TGFbeta2mediated EMT of lens epithelial cells: implications for age-associated fibrosis. Aging Cell 15, 465-476 (2016).

48. Zhang, P., Dai, H. \& Peng, L. AGEs induce epithelial to mesenchymal transformation of human peritoneal mesothelial cells via upregulation of STAT3. Glycoconj. J. 36, 155-163 (2019).
49. Ma, J., Liu, T. \& Dong, X. Advanced glycation end products of bovine serum albumin-induced endothelial-to-mesenchymal transition in cultured human and monkey endothelial cells via protein kinase B signaling cascades. Mol. Vis. 16, 2669-2679 (2010).

50. De Meyer, G. R. et al. Autophagy in vascular disease. Circ. Res. 116, 468-479 (2015).

51. Platini, F., Perez-Tomas, R., Ambrosio, S. \& Tessitore, L. Understanding autophagy in cell death control. Curr. Pharm. Des. 16, 101-113 (2010).

52. Alva, A. S., Gultekin, S. H. \& Baehrecke, E. H. Autophagy in human tumors: cell survival or death? Cell Death Differ. 11, 1046-1048 (2004).

53. Levine, B. \& Kroemer, G. Autophagy in the pathogenesis of disease. Cell 132, 27-42 (2008).

54. Gustafsson, A. B. \& Gottlieb, R. A. Autophagy in ischemic heart disease. Circ. Res. 104, 150-158 (2009).

55. Orogo, A. M. \& Gustafsson, A. B. Therapeutic targeting of autophagy: potential and concerns in treating cardiovascular disease. Circ. Res. 116, 489-503 (2015).

56. Chen, X., Xu, S., Zhao, C. \& Liu, B. Role of TLR4/NADPH oxidase 4 pathway in promoting cell death through autophagy and ferroptosis during heart failure. Biochem. Biophys. Res. Commun. 516, 37-43 (2019).

57. Zhang, X. Effect of typhaneoside on ventricular remodeling and regulation of PI3K/Akt/mTOR pathway. Herz 45, 113-122 (2020).

58. Patschan, S. et al. Lipid mediators of autophagy in stress-induced premature senescence of endothelial cells. Am. J. Physiol. Heart C. 294, H1119-H1129 (2008). 